\title{
The effects of CRF and urocortins on the hippocampal glutamate release
}

\author{
Zsolt Bagosi*, Beáta Balangó, Dávid Pintér, Krisztina Csabafi, Miklós Jászberényi, \\ Gyula Szabó, Gyula Telegdy \\ Department of Pathophysiology, Faculty of Medicine, University of Szeged, Hungary
}

\section{A R T I C L E I N F O}

\section{Article history:}

Received 20 May 2015

Received in revised form

3 July 2015

Accepted 19 July 2015

Available online 26 July 2015

\section{Keywords:}

Superfusion

CRF

Urocortin

Glutamate

HPA axis

\begin{abstract}
A B S T R A C T
Corticotropin-releasing factor (CRF) is a hypothalamic neurohormone and an extrahypothalamic neurotransmitter that regulates the hypothalamic-pituitary-adrenal (HPA) axis. The urocortins (UCN I, UCN II and UCN III) are CRF-related peptides, which may also regulate the HPA axis directly or indirectly, by modulation of extrahypothalamic neurotransmitters, such as amygdalar GABA and hippocampal glutamate.

Our previous in vitro superfusion studies have already demonstrated that CRF and UCN I stimulate the amygdalar GABA release in rats. The aim of the present study was to investigate the effects of CRF, UCN I, UCN II and UCN III on the glutamate release elicited electrically from rat hippocampal slices in similar in vitro conditions. In order to investigate the participation of CRF receptors (CRFR1 and CRFR2) in this process, hippocampal slices were pretreated with antalarmin, a selective antagonist of CRFR1 or astressin 2B, a selective antagonist of CRFR2.

CRF and UCN I at $100 \mathrm{nM}$ decreased significantly the hippocampal glutamate release evoked by electrical stimulation. In contrast, 100 nM of UCN II and UCN III did not affect significantly the hippocampal glutamate release enhanced by electrical stimulation. The decreasing effects of CRF and UCN I were reversed by antalarmin, but not by astressin 2B, both being administered in equimolar doses.

Our results demonstrate that CRF and UCN I inhibit the glutamate release in the hippocampus via CRFR1 and that CRFR2 does not participate to this process. Based on the previous and the present results we conclude that CRFR1 agonists can activate the HPA axis not only directly, but also indirectly by increasing the amygdalar GABA release and decreasing the hippocampal glutamate release.
\end{abstract}

(C) 2015 Elsevier Ltd. All rights reserved.

\section{Introduction}

Corticotropin-releasing factor (CRF) is a hypothalamic neurohormone and an extrahypothalamic neurotransmitter that mediates the endocrine, autonomic and behavioral responses to stress (Vale et al., 1981). As a hypothalamic neurohormone, CRF activates the hypothalamic-pituitary-adrenal (HPA) axis (Carrasco and Van de Kar, 2003). CRF is secreted from the paraventricular nucleus (PVN) of the hypothalamus and released into circulation at the level of median eminence; reaching the anterior pituitary it stimulates the secretion of adrenocorticotrop hormone (ACTH), which on its turn stimulates the production of glucocorticoids in the adrenal

\footnotetext{
* Corresponding author. Department of Pathophysiology, University of Szeged, 6725, Szeged, Semmelweis Str. 1, Hungary.

E-mail address: bagosi.zsolt@med.u-szeged.hu (Z. Bagosi).
}

cortex (Tsigos and Chrousos, 2002). The increase of plasma glucocorticoid concentration not only reflects the activation of the HPA axis, but it exerts negative feedback effects on the hypothalamus, the anterior pituitary and the hippocampus and positive feedback effect on the amygdala (Herman and Cullinan, 1997).

As an extrahypothalamic neurotransmitter, CRF may also modulate the HPA axis (Herman and Cullinan, 1997). CRF is also synthesized in the central nucleus of the amygdala ( $\mathrm{CeA}$ ) found in the vicinity of the medial nucleus of the amygdala (MeA) and the ventral subiculum (vSub) region of the hippocampus (Reul and Holsboer, 2002). Neurons from these regions (especially Mea and vSub) send GABAergic or glutamatergic projections to the GABAergic neurons of the bed nucleus of the stria terminalis (BNST) and the peri-paraventricular nuclei (peri-PVN), which exert a tonic GABAergic inhibition upon the paraventricular CRF synthesis/ release (Bale and Vale, 2004; Cullinan et al., 2008). Thus, the 
amygdala through GABAergic-GABAergic disinhibition increases, whereas the hippocampus through glutamatergic-GABAergic inhibition decreases the activity of the HPA axis, respectively (Herman et al., 2003, 2004).

CRF acts through two distinct receptors, CRFR1 and CRFR2, which seem to have antagonistic effects in the brain (Chang et al., 1993; Van Pett et al., 2000). Activation of CRFR1 induces HPA axis activation, anxiety and depression, while activation of CRFR2 produces anxiolytic and antidepressive effects (Bale and Vale, 2004; Reul and Holsboer, 2002). However, our recent studies suggest that the role of CRFR2 in the regulation of the HPA axis can be inhibitory or stimulatory, depending on the actual concentration of their agonists, the urocortins (Bagosi et al., 2013, 2014). The urocortins (UCN I, UCN II and UCN III) are CRF-related peptides, with similar chemical structure, but different pharmacological profile, than that of CRF (Fekete and Zorrilla, 2007; Suda et al., 2004). In contrast with CRF, which binds preferentially to CRFR1, UCN I has equal affinity for both CRF receptors (Vaughan et al., 1995). UCN II and UCN III bind selectively to CRFR2 (Lewis et al., 2001; Reyes et al., 2001).

Our previous in vitro superfusion experiments have demonstrated that CRF and UCN I stimulate the amygdalar GABA release in rats and that this stimulatory effect is mediated via CRFR1, and not CRFR2 (Bagosi et al., 2008). The aim of the present experiments was to investigate the effects of CRF and urocortins on the hippocampal glutamate release in rats in similar in vitro conditions.

\section{Materials and methods}

Male Wistar rats (Animal Husbandry Services, Domaszék, Hungary) weighing 150-250 g were used. During the experiments they were kept and handled in accordance with the instructions of the University of Szeged Ethical Committee for the Protection of Animals in Research which are concordant with the European Communities Council Directive of 24 November 1986 (86/609/EEC). The rats were decapitated and their brains were rapidly removed. All efforts were made to minimize animal suffering and to reduce the number of animals used ( $\mathrm{N}=2$ for each experiment). The hippocampus was isolated and dissected in a Petri dish filled with ice-cold Krebs solution (composition: $113 \mathrm{mM} \mathrm{NaCl}, 4.7 \mathrm{mM} \mathrm{KCl}$, $1.2 \mathrm{mM} \mathrm{MgSO}_{4}, 25 \mathrm{mM} \mathrm{NaHCO}_{3}, 11.5 \mathrm{mM}$ glucose, $1.2 \mathrm{mM} \mathrm{KH}_{2} \mathrm{PO}_{4}$, $2.5 \mathrm{mM} \mathrm{CaCl}_{2}$, pH 7.4; Reanal, Hungary) according to the Stereotaxic Atlas Of The Rat Brain (Pellegrino et al., 1979). The extracted hippocampus was cut with a Mcllwain tissue chopper and slices of $300 \mu \mathrm{M}$ were produced.

The hippocampal slices were investigated with in vitro superfusion method described originally by Gaddum (Gaddum, 1953). The slices were incubated for $30 \mathrm{~min}$ in $8 \mathrm{ml}$ of Krebs solution, submerged in a water bath at $37^{\circ} \mathrm{C}$ and gassed through a single-use needle with a mixture of $5 \% \mathrm{CO}_{2}$ and $95 \% \mathrm{O}_{2}$. During the incubation $\left[{ }^{3} \mathrm{H}\right]$ glutamate (Biotrend Ltd., Germany) at $20 \mu \mathrm{M}$ with a specific activity of $40 \mathrm{Ci} / \mathrm{mmol}$ was injected into the incubation medium. Two tritiated slices were transferred to each of the four cylindrical perspex chambers of the superfusion system (Experimetria Ltd, Hungary). A multichannel peristaltic pump (Gilson Minipuls 2, USA) was used to maintain a constant superfusion rate of $200 \mu \mathrm{l} /$ min. The slices were superfused for 30 min to allow tissue equilibrium. In order to determine the effects of different CRFR agonists on the hippocampal glutamate release equimolar doses (100 nM) of CRF, UCN I, UCN II or UCN III (Bachem Ltd., Germany) were added to the perfusion medium, 20 min after the superfusion had started. In order to determine the participation of CRF receptors in this process $100 \mathrm{nM}$ of selective CRFR1 antagonist antalarmin or $100 \mathrm{nM}$ of selective CRFR2 antagonist astressin-2B (Sigma-Aldrich Inc., USA) were preadded to the perfusion medium, $10 \mathrm{~min}$ after the superfusion had started. The concentrations of the non-selective agonists were selected based on preliminary experiments in which $100 \mathrm{nM}$ of CRF and $100 \mathrm{nM}$ UCN I proved the most effective in stimulating the hippocampal glutamate release. Using equimolar concentrations of the selective agonists/antagonists was inspired by our previous studies investigating the amygdalar and the hypothalamic GABA release (Bagosi et al., 2008, 2012).

The superfusates were collected in Eppendorf tubes by a multichannel fraction collector (Gilson FC 203B, UK) every 2 min. After the first 2 min electrical stimulation consisting of squarewave impulses (total duration: $2 \mathrm{~min}$, voltage: $100 \mathrm{~V}$, pulse length: $5 \mathrm{~ms}$, frequency: $10 \mathrm{~Hz}$ ) was delivered to each of the four chambers, as gold electrodes were previously attached to both halves of the superfusion chambers and connected to an ST-02 electrical stimulator (Experimetria Ltd., Hungary). The fraction collecting lasted $32 \mathrm{~min}$, thus 16 fractions were obtained.

After the fraction collecting had finished, $3 \mathrm{ml}$ of scintillation fluid (Ultima Gold, Perkin-Elmer Inc., USA) were added to each fraction and the remnants of the superfused brain slices, which were previously solubilized in $200 \mathrm{ml}$ of Krebs solution using an ultrasonic homogenizer (Branson Sonifier 250, USA). The radioactivity in the fractions and the homogenized tissue samples was measured with a liquid scintillation spectrometer (Tri-carb 2100 TR, Packard Inc., USA). The fractional release was calculated as a percentage of the radioactivity present in the collected sample compared to the total radioactivity of the corresponding tissue.

The fractional release of glutamate was calculated by the areaunder-the-curve (AUC) method and statistical analysis of the results was performed by analysis of variance (SigmaPlot v11.0, Systat Software Inc., USA). Differences between two areas were determined by Student's t test or two-way ANOVA followed by Tukey post-hoc test and a probability level of 0.05 or less was accepted as indicating a statistically significant difference.

\section{Results}

CRF and UCN I (both of $100 \mathrm{nM}$ concentration) decreased significantly the hippocampal $\left[{ }^{3} \mathrm{H}\right]$ glutamate release elicited by electrical stimulation $[\mathrm{t}(6,11)=2.816 ; \mathrm{p}<0.05$ for CRF $v$ s. the control, and $\mathrm{t}(6,11)=3.352 ; \mathrm{p}<0.05$ or UCN I $v$. the control] (Figs. 1 and 2). In contrast, UCN II and UCN III (both of $100 \mathrm{nM}$ concentration) did not affect significantly the hippocampal $\left[{ }^{3} \mathrm{H}\right]$

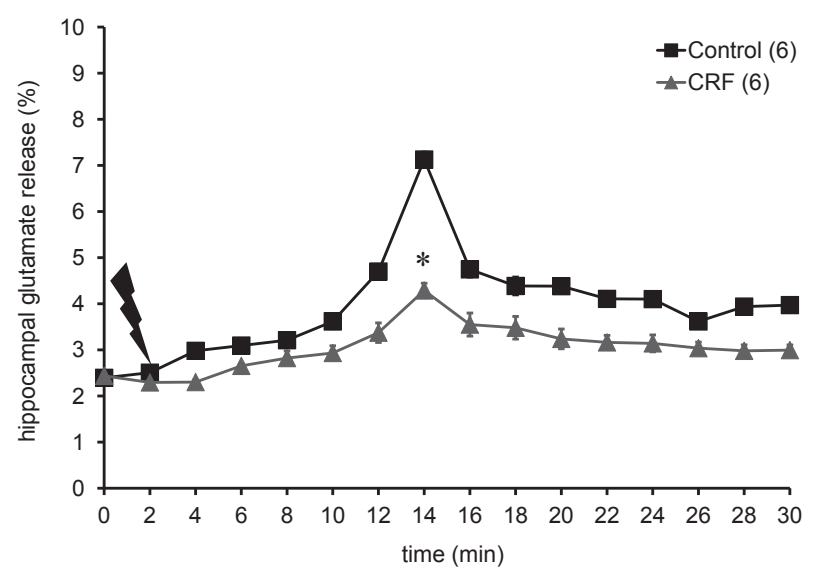

Fig. 1. The effects of CRF on the hippocampal glutamate release in rats. CRF (100 nM) decreased significantly the fractional $\left[{ }^{3} \mathrm{H}\right]$ glutamate release from rat hippocampal slices following electrical stimulation. Values are presented as fractional release (\%) \pm SEM; the number of animals used was 2 for each experiment and the numbers of slices is indicated in brackets. A probability level of 0.05 or less was accepted as a statistically significant difference and indicated with ${ }^{*} \mathrm{p}<0.05$ for CRF vs. control. 


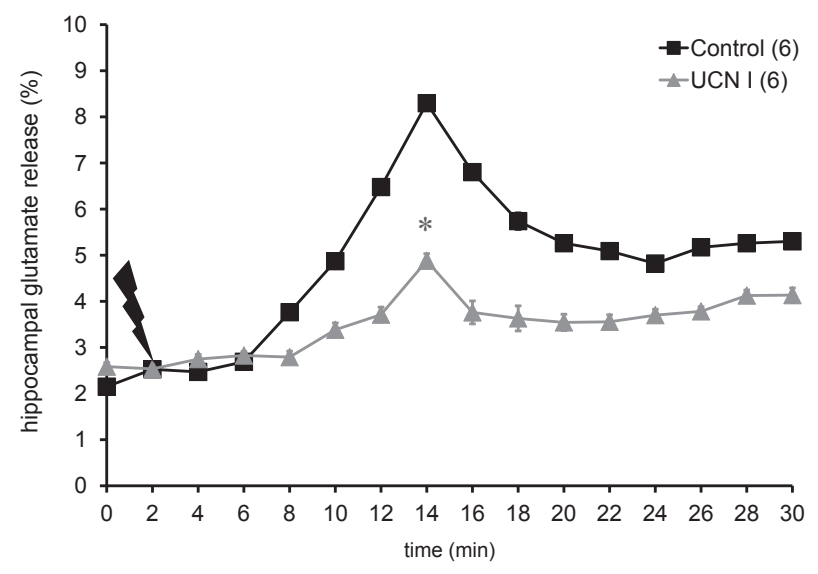

Fig. 2. The effects of UCN I on the hippocampal glutamate release in rats. UCN I $(100 \mathrm{nM})$ decreased significantly the fractional $\left[{ }^{3} \mathrm{H}\right]$ glutamate release from rat hippocampal slices following electrical stimulation. Values are presented as fractional release $(\%) \pm S E M$; the number of animals used was 2 for each experiment and the numbers of slices is indicated in brackets. A probability level of 0.05 or less was accepted as a statistically significant difference and indicated with ${ }^{*} \mathrm{p}<0.05$ for UCN I vs. control.

glutamate release enhanced by electrical stimulation (Figs. 3 and 4 ). The effect of $\operatorname{CRF}[\mathrm{F}(2,31)=5.303 ; \mathrm{p}<0.05$ for $\mathrm{CRF} v$ s. control] was reversed remarkably by antalarmin $[\mathrm{F}(1,31)=2.387$; $\mathrm{p}<0.05$ for $\mathrm{CRF}+$ antalarmin $v s$. CRF alone], but not by astressin $2 \mathrm{~B}$, both being administered in equimolar doses (100 nM). Also, the effect of UCN I $[\mathrm{F}(2,31)=15.712 ; \mathrm{p}<0.05$ for UCN I + antalarmin $v s$. UCN I alone $]$ was reversed completely by antalarmin $[\mathrm{F}(1,31)=13.958 ; \mathrm{p}<0.05$ for UCN I + antalarmin vs. UCN I alone], but not by astressin $2 \mathrm{~B}$, both being administered in equimolar doses (100 nM). CRF, UCN I, UCN II or UCN III did not change the basal release of $\left[{ }^{3} \mathrm{H}\right]$ glutamate. Nevertheless, antalarmin and astressin 2B alone, did not change the stimulated release of $\left[{ }^{3} \mathrm{H}\right]$ glutamate (Figs. 5 and 6).

\section{Discussion}

Our results demonstrate that of CRF and UCN I inhibit the glutamate release in the hippocampus via CRFR1, as the selective CRFR1 antagonist was able to increase the hippocampal glutamate

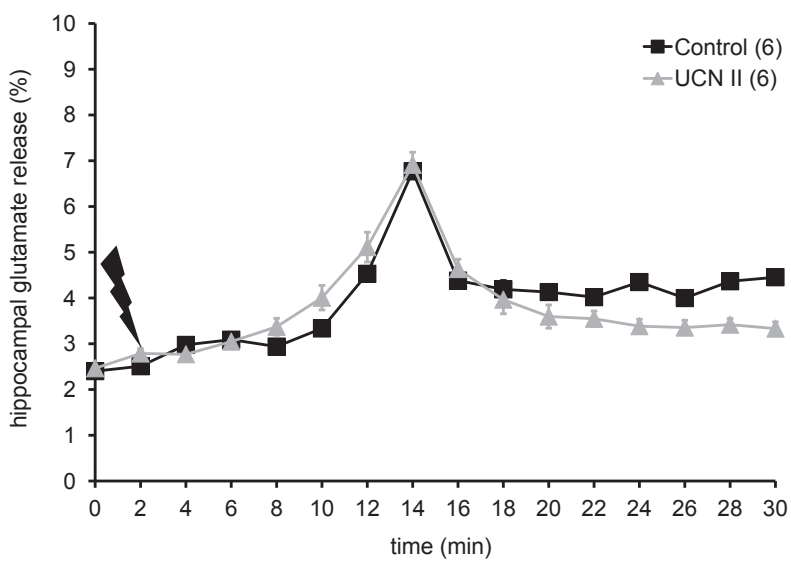

Fig. 3. The effects of UCN II on the hippocampal glutamate release in rats. UCN II $(100 \mathrm{nM})$ did not influence significantly the fractional $\left[{ }^{3} \mathrm{H}\right]$ glutamate release from rat hippocampal slices following electrical stimulation. Values are presented as fractional release $(\%) \pm S E M$; the number of animals used was 2 for each experiment and the numbers of slices is indicated in brackets. A probability level of 0.05 or less was accepted as a statistically significant difference.

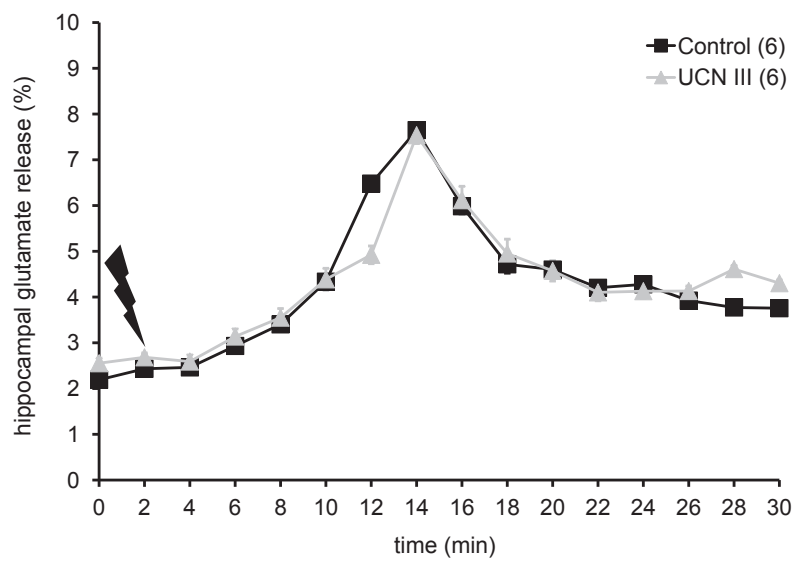

Fig. 4. The effects of UCN III on the hippocampal glutamate release in rats. UCN IIl $(100 \mathrm{nM})$ did not influence significantly the fractional $\left[{ }^{3} \mathrm{H}\right]$ glutamate release from rat hippocampal slices following electrical stimulation. Values are presented as fractional release $(\%) \pm S E M$; the number of animals used was 2 for each experiment and the numbers of slices is indicated in brackets. A probability level of 0.05 or less was accepted as a statistically significant difference.

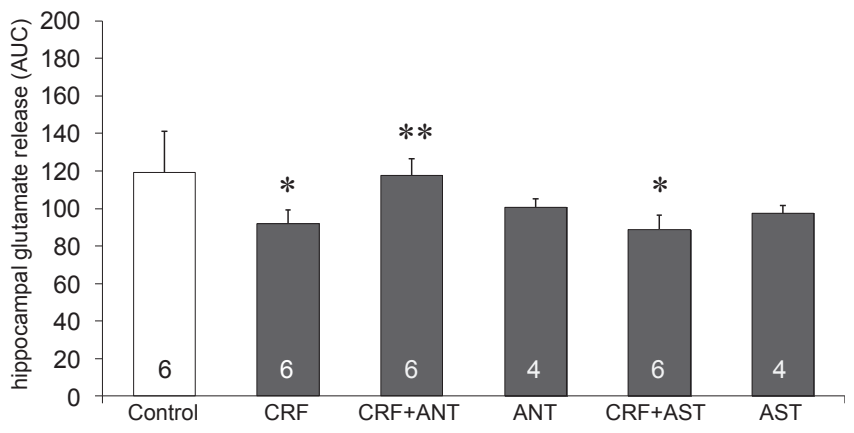

Fig. 5. The effects of antalarmin (ANT) and astressin 2B (AST) on the hippocampal glutamate release decreased by CRF in rats. Antalarmin $(100 \mathrm{nM})$ increased remarkably the fractional $\left[{ }^{3} \mathrm{H}\right]$ glutamate release decreased by CRF (100 nM) from rat hippocampal slices following electrical stimulation, but astressin $2 \mathrm{~B}(100 \mathrm{nM})$ did not change it considerably. Antalarmin and astressin $2 \mathrm{~B}$ alone were ineffective. Values are presented as area-under-the-curve (AUC) \pm SEM; the number of animals used was 2 for each experiment and the numbers of slices is indicated on the columns. A probability level of 0.05 or less was accepted as a statistically significant difference and indicated with * $\mathrm{p}<0.05$ for treatment $v$ s. control and ${ }^{* *} \mathrm{p}<0.05$ for CRF + treatment $v$ s. CRF alone.

release decreased previously by the non-selective CRFR1 agonists. CRFR2 is not involved in this process, since the selective CRFR2 antagonist did not reverse the effects of CRF or UCN I and the selective CRFR2 agonists UCN II and UCN III did not affect the hippocampal glutamate release either.

Our previous in vitro superfusion study reported that CRF and UCN I stimulate the amygdalar GABA release via CRFR1, but not CRFR2 (Bagosi et al., 2008). We speculated that this amygdalar GABA is released from both the MeA and the CeA, which respond to distinct stressors and are thought to have divergent roles in HPA regulation. Neurons from the MeA are activated following exposure to emotional stressors including predator, social interaction, forced swimming and restraint stress paradigms and send mainly GABAergic projections to GABAergic neurons of BNST and the periPVN which directly innervate the PVN leading to activation actually to disinhibition - of the HPA axis (Smith and Vale, 2006). In contrast, the $\mathrm{CeA}$ is activated following exposure to homeostatic stressors, including hemorrhage and immune challenge and exerts its feed-forward effect on the HPA axis through interneurons localized in the brain stem (Smith and Vale, 2006). 


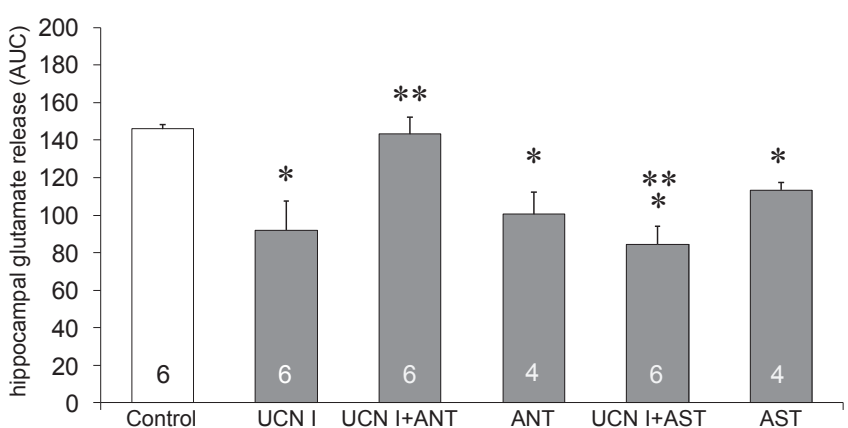

Fig. 6. The effects of antalarmin (ANT) and astressin $2 \mathrm{~B}$ (AST) on the hippocampal glutamate release decreased by urocortin I (UCN I) in rats. Antalarmin (100 nM) reversed completely the fractional $\left[{ }^{3} \mathrm{H}\right]$ glutamate release decreased by UCN I (100 nM) from rat hippocampal slices following electrical stimulation, but astressin $2 \mathrm{~B}(100 \mathrm{nM})$ did not change it considerably. Antalarmin and astressin $2 \mathrm{~B}$ alone were ineffective. Values are presented as area-under-the-curve $(A U C) \pm S E M$; the number of animals used was 2 for each experiment and the numbers of slices is indicated on the columns. A probability level of 0.05 or less was accepted as a statistically significant difference and indicated with ${ }^{*} \mathrm{p}<0.05$ for treatment $v$ s. control ${ }^{* *} \mathrm{p}<0.05$ for UCN I + treatment vs. UCN I alone.

The present in vitro superfusion study completes our previous report with the observation that CRF and UCN I inhibit the hippocampal glutamate release via CRFR1, and not CRFR2. We presume that a similar process may occur under different stressors in the

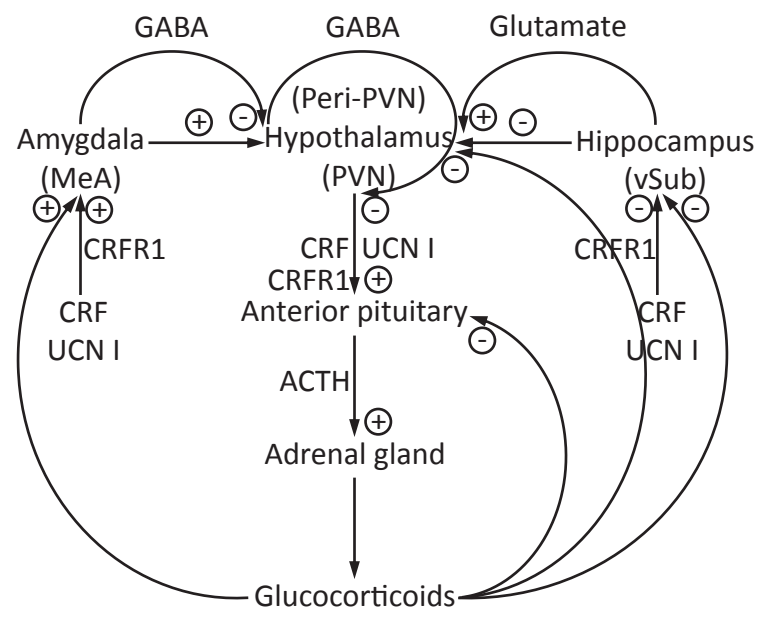

Fig. 7. The effects of CRF and urocortin I (UCN I) on the amygdalar GABA and the hippocampal glutamate release and their putative impacts on the hypothalamicpituitary-adrenal (HPA) axis. CRF is secreted from the paraventricular nucleus (PVN) of the hypothalamus and released into circulation at the level of median eminence; reaching the anterior pituitary it stimulates the secretion of adrenocorticotrop hormone $(\mathrm{ACTH})$, which on its turn stimulates the production of glucocorticoids in the adrenal cortex. The increase of plasma glucocorticoid concentration not only reflects the activation of the HPA axis, but it exerts negative feedback effects on the hypothalamus, the anterior pituitary and the hippocampus and positive feedback effect on the amygdala. CRF is also synthesized in the central nucleus of the amygdala (CeA) found in the vicinity of the medial nucleus of the amygdala (MeA) and the ventral subiculum (vSub) region of the hippocampus. Neurons from these regions (especially MeA and vSub) send GABAergic or glutamatergic projections to the GABAergic neurons of the peri-paraventricular nuclei (peri-PVN), which exert a tonic inhibition upon the paraventricular CRF synthesis/release. Thus, the amygdala through GABAergic-GABAergic disinhibition increases, whereas the hippocampus through glutamatergic-GABAergic inhibition decreases the activity of the HPA axis, respectively. Based on the previous and the present results we propose that CRFR1 agonists can activate the HPA axis not only directly by stimulating the pituitary ACTH (via CRFR1) and consequently the adrenal glucocorticoid secretion, but also indirectly by increasing the amygdalar GABA release (via CRFR1) and decreasing the hippocampal glutamate release (via CRFR1).
vSub region of the hippocampus, which has been implicated in the regulation of the HPA axis. Hippocampal lesions involving the vSub were shown to produce exaggerated HPA responses to restraint and open field exposure, but not to hypoxia or ether exposure, suggesting that hippocampal neurons respond to distinct stress modalities (Smith and Vale, 2006). Neurons from this region send mostly glutamatergic projections to GABAergic neurons of BNST and the peri-PVN which directly innervate the PVN, resulting ultimately in inhibition of the HPA axis (Smith and Vale, 2006).

The interaction of CRF and urocortins with glutamate have been investigated in other in vitro settings also. An earlier study indicated that CRF and UCN I modulate differently the excitatory glutamatergic synaptic transmission in the CeA and the lateral septum, which are reciprocally innervated (Liu et al., 2004). Another study concluded that UCN I, but not UCN II, protects cultured hippocampal neurons from oxidative stress and glutamatergic excitotoxicity via CRFR1, even more potently than CRF does (Pedersen et al., 2002). We suggest that besides having role in neurotransmission and neuroprotection, CRF-glutamate and UCN I-glutamate interactions may also take part in the regulation of the HPA axis. Based on the previous and the present results, we propose that CRFR1 agonists can activate the HPA axis not only directly by stimulating the pituitary ACTH and consequently the adrenal glucocorticoid secretion, but also indirectly by increasing the amygdalar GABA release and decreasing the hippocampal glutamate release (Fig. 7).

\section{Acknowledgments}

This work was supported by the following grants: TÁMOP 4.2.2A-11/KONV-2012-0052, KTIA_13_NAP-A-III/8.

\section{References}

Bagosi, Z., Csabafi, K., Jaszberenyi, M., Telegdy, G., 2012. The effects of corticotropinreleasing factor and the urocortins on hypothalamic gamma-amino butyric acid release-the impacts on the hypothalamic-pituitary-adrenal axis. Neurochem. Int. 60, 350-354

Bagosi, Z., Csabafi, K., Palotai, M., Jaszberenyi, M., Foldesi, I., Gardi, J., Szabo, G., Telegdy, G., 2013. The interaction of Urocortin II and Urocortin III with amygdalar and hypothalamic cotricotropin-releasing factor (CRF)-reflections on the regulation of the hypothalamic-pituitary-adrenal (HPA) axis. Neuropeptides 47, $333-338$.

Bagosi, Z., Csabafi, K., Palotai, M., Jaszberenyi, M., Foldesi, I., Gardi, J., Szabo, G., Telegdy, G., 2014. The effect of urocortin I on the hypothalamic ACTH secretagogues and its impact on the hypothalamic-pituitary-adrenal axis. Neuropeptides 48, 15-20.

Bagosi, Z., Jaszberenyi, M., Szabo, G., Telegdy, G., 2008. The effects of CRF and the urocortins on $\left[{ }^{3} \mathrm{H}\right] \mathrm{GABA}$ release from the rat amygdala-an in vitro superfusion study. Brain Res. Bull. 75, 15-17.

Bale, T.L., Vale, W.W., 2004. CRF and CRF receptors: role in stress responsivity and other behaviors. Annu. Rev. Pharmacol. Toxicol. 44, 525-557.

Carrasco, G.A., Van de Kar, L.D., 2003. Neuroendocrine pharmacology of stress. Eur. J. Pharmacol. 463, 235-272.

Chang, C.P., Pearse 2nd, R.V., O'Connell, S., Rosenfeld, M.G., 1993. Identification of a seven transmembrane helix receptor for corticotropin-releasing factor and sauvagine in mammalian brain. Neuron 11, 1187-1195.

Cullinan, W.E., Ziegler, D.R., Herman, J.P., 2008. Functional role of local GABAergic influences on the HPA axis. Brain Struct. Funct. 213, 63-72.

Fekete, E.M., Zorrilla, E.P., 2007. Physiology, pharmacology, and therapeutic relevance of urocortins in mammals: ancient CRF paralogs. Front. Neuroendocrinol. $28,1-27$.

Gaddum, J.H., 1953. The technique of superfusion. Br. J. Pharmacol. Chemother. 8, $321-326$.

Herman, J.P., Cullinan, W.E., 1997. Neurocircuitry of stress: central control of the hypothalamo-pituitary-adrenocortical axis. Trends Neurosci. 20, 78-84.

Herman, J.P., Figueiredo, H., Mueller, N.K., Ulrich-Lai, Y., Ostrander, M.M., Choi, D.C. Cullinan, W.E., 2003. Central mechanisms of stress integration: hierarchical circuitry controlling hypothalamo-pituitary-adrenocortical responsiveness. Front. Neuroendocrinol. 24, 151-180.

Herman, J.P., Mueller, N.K., Figueiredo, H., 2004. Role of GABA and glutamate circuitry in hypothalamo-pituitary-adrenocortical stress integration. Ann. N. Y. Acad. Sci. 1018, 35-45.

Lewis, K., Li, C., Perrin, M.H., Blount, A., Kunitake, K., Donaldson, C., Vaughan, J., 
Reyes, T.M., Gulyas, J., Fischer, W., Bilezikjian, L., Rivier, J., Sawchenko, P.E., Vale, W.W., 2001. Identification of urocortin III, an additional member of the corticotropin-releasing factor (CRF) family with high affinity for the CRF2 receptor. Proc. Natl. Acad. Sci. U. S. A. 98, 7570-7575.

Liu, J., Yu, B., Neugebauer, V., Grigoriadis, D.E., Rivier, J., Vale, W.W., ShinnickGallagher, P., Gallagher, J.P., 2004. Corticotropin-releasing factor and Urocortin I modulate excitatory glutamatergic synaptic transmission. J. Neurosci. 24, 4020-4029.

Pedersen, W.A., Wan, R., Zhang, P., Mattson, M.P., 2002. Urocortin, but not urocortin II, protects cultured hippocampal neurons from oxidative and excitotoxic cell death via corticotropin-releasing hormone receptor type I. J. Neurosci. 22, 404-412.

Pellegrino, L.J., Pellegrino, A.S., Cushman, A.J., 1979. A Stereotaxic Atlas of the Rat Brain, second ed. Plenum Press, New York.

Reul, J.M., Holsboer, F., 2002. Corticotropin-releasing factor receptors 1 and 2 in anxiety and depression. Curr. Opin. Pharmacol. 2, 23-33.

Reyes, T.M., Lewis, K., Perrin, M.H., Kunitake, K.S., Vaughan, J., Arias, C.A Hogenesch, J.B., Gulyas, J., Rivier, J., Vale, W.W., Sawchenko, P.E., 2001. Urocortin II: a member of the corticotropin-releasing factor (CRF) neuropeptide family that is selectively bound by type 2 CRF receptors. Proc. Natl. Acad. Sci. U. S. A. 98
$2843-2848$

Smith, S.M., Vale, W.W., 2006. The role of the hypothalamic-pituitary-adrenal axis in neuroendocrine responses to stress. Dialogues Clin. Neurosci. 8, 383-395.

Suda, T., Kageyama, K., Sakihara, S., Nigawara, T., 2004. Physiological roles of urocortins, human homologues of fish urotensin I, and their receptors. Peptides 25, 1689-1701.

Tsigos, C., Chrousos, G.P., 2002. Hypothalamic-pituitary-adrenal axis, neuroendocrine factors and stress. J. Psychosom. Res. 53, 865-871.

Vale, W., Spiess, J., Rivier, C., Rivier, J., 1981. Characterization of a 41-residue ovine hypothalamic peptide that stimulates secretion of corticotropin and betaendorphin. Science 213, 1394-1397.

Van Pett, K., Viau, V., Bittencourt, J.C., Chan, R.K., Li, H.Y., Arias, C., Prins, G.S. Perrin, M., Vale, W., Sawchenko, P.E., 2000. Distribution of mRNAs encoding CRF receptors in brain and pituitary of rat and mouse. J. Comp. Neurol. 428, $191-212$.

Vaughan, J., Donaldson, C., Bittencourt, J., Perrin, M.H., Lewis, K., Sutton, S., Chan, R. Turnbull, A.V., Lovejoy, D., Rivier, C., et al., 1995. Urocortin, a mammalian neuropeptide related to fish urotensin I and to corticotropin-releasing factor. Nature 378, 287-292. 